\title{
Lenard und Einstein. Ihr Briefwechsel und ihr Verhältnis vor der Nauheimer Diskussion von 1920
}

Von Andreas Kleinert und Charlotte Schönbeck

Über Lenards Kampf gegen Einstein in den zwanziger Jahren ist in Veröffentlichungen zur Physikgeschichte des 20. Jahrhunderts und in Einstein-Biographien viel geschrieben worden ${ }^{1}$. Bekanntlich nahmen diese Auseinandersetzungen durch die Verquickung von fachlicher Kontroverse, persönlichen Angriffen und antisemitischer Propaganda eine für wissenschaftliche Dispute durchaus ungewöhnliche Dimension an. Der in der breiten Öffentlichkeit geführte Kampf begann 1920, als sich Lenard und Einstein in Bad Nauheim in einem Rededuell gegenüberstanden, und endete mit dem oft zitierten Artikel im «Völkischen Beobachter» vom 13.5.1933, in dem Lenard seiner Genugtuung Ausdruck gibt, daß der «Relativitätsjude», dessen «mathematisch zusammengestoppelte 'Theorie' (...) nun schon allmählich in Stücke zerfällt», Deutschland verlassen hat. Die Vorgeschichte dieser Feindschaft, insbesondere der von gegenseitiger Hochachtung geprägte Briefwechsel zwischen den beiden Gelehrten, ist jedoch bisher, von einigen Andeutungen abgesehen ${ }^{2}$, unbekannt geblieben; davon soll im folgenden die Rede sein.

Als Einstein im Jahre 1905 die drei Arbeiten veröffentlichte, die seinen späteren Ruhm begründen sollten, war er in der wissenschaftlichen Welt unbekannt. Auch für Lenard, seit 1898 Ordinarius an der Universität Kiel, war der technische Experte 3.Klasse am Amt für geistiges Eigentum in Bern damals sicher ein Unbekannter. Einstein hingegen war mit den Arbeiten des 17 Jahre älteren Lenard durchaus vertraut: in dem Artikel «Über einen die Erzeugung und Verwandlung des Lichtes betreffenden heuristischen Gesichtspunkt» (Deutung des Photoeffekts mit Hilfe der Lichtquantenhypothese) bezieht er sich mehrfach auf Lenards Untersuchungen aus den Jahren 1902-1903, und zu der von ihm postulierten Proportionalität von Elektronenenergie und Frequenz des einfallenden Lichtes schreibt er ausdrücklich: «Mit den von Hrn. Lenard beobachteten Eigenschaften der lichtelektrischen Wirkung steht unsere Auffassung, soweit ich sehe, nicht im Widerspruch. » ${ }^{3}$

Es ist nicht bekannt, ob Einstein ein Exemplar dieser Arbeit an Lenard geschickt hat oder ob Lenard von sich aus bei der Lektüre der «Annalen der Physik» darauf gestoßen ist; jedenfalls wurde er auf Einstein aufmerksam und schickte ihm wenig später eine seiner neueren Arbeiten zu. Dafür bedankte sich Einstein mit dem folgenden Brief: 
Hochgeehrter Herr Professor!

Bern den 16. November 1905.

Ich danke Ihnen herzlich für die mir gesandte Arbeit, die ich mit demselben Gefühl der Bewunderung studierte wie Ihre früheren Arbeiten. Bei dieser Gelegenheit möchte ich mir eine kurze sachliche Bemerkung erlauben.

Die mir bekannten Experimente schliessen die Möglichkeit nicht aus, dass die Emission bezw. Absorption jeder einzelnen Spektrallinie an einen bestimmten, für sie charakteristischen Zustand des emittirenden bezw. absorbirenden Zentrums (Atoms) gebunden sei. Analoges haben Sie ja bereits für die einzelnen Serien bewiesen. A priori spräche die Einfachheit für eine derartige Annahme, da man für die Lichtemission bezw. -absorption nicht Mechanismen annehmen müsste, welche die Mannigfaltigkeit einer ganzen Serie besitzen.

Nach der angedeuteten Auffassung wäre die Absorption einer Serie durch einen (kalten) Dampf so aufzufassen, dass die Absorption von Licht der Linie $v_{1}$ das betreffende absorbirende Zentrum aufnahmefähig macht für Licht von der Linie $v_{2}$ etc. Es wäre dann eine Absorption von $v_{2}$ nur bei gleichzeitiger Absorption von $v_{1}$ durch den Dampf möglich.

Mit vorzüglicher Hochachtung

A. Einstein

Bei der Arbeit, für die Einstein hier dankt, handelt es sich offenbar um Lenards Aufsatz «Über Lichtemission der Alkalidämpfe und Salze, und über die Zentren dieser Emissionen», erschienen in Heft 7 desselben 17. Bandes der «Annalen der Physik» von 1905, in dem auch Einsteins Arbeit erschienen war ${ }^{4}$. Einsteins Bemerkung knüpft nämlich an die Deutung der Versuche an, die in dieser Arbeit Lenards behandelt werden.

Ein zentrales Thema der Physik jener Zeit war die Frage nach der Struktur des Atoms. Die Untersuchung von Spektren sollte die Entstehung der Spektrallinien und den dieser Erscheinung zugrunde liegenden Mechanismus klären ${ }^{5}$. Lenard hatte schon 1903 vermutet, daß ein Atom nicht gleichzeitig alle Linien emittieren kann, sondern «daß jedes Metallatom (...) eine Reihe verschiedener Zustände annehme, deren mindestens so viele sind, als ein Spektrum Serien enthält. ${ }^{6}$ In der Arbeit von 1905 erweiterte er diese Hypothese dann durch die zusätzliche Annahme, Ursachen der Hauptserien seien verschiedene Zustände der elektrisch neutralen Atome, während die Nebenserien durch ein- oder mehrfach ionisierte Atome zustande kommen sollten. An diese Vermutung Lenards knüpft Einstein in seinem Brief an, wenn er vorschlägt, nicht nur jeder Serie, sondern jeder Linie des Spektrums einen besonderen Zustand des Atoms zuzuschreiben. Es sollte noch über 20 Jahre dauern, bis eine befriedigende Erklärung dieser Erscheinungen durch die Quantenmechanik möglich wurde.

Lenard hat Einsteins Brief zunächst nicht beantwortet. Es gab jedoch ab 1908 eine indirekte Verbindung zwischen Lenard und Einstein durch den Physiker 
Jakob Johann Laub. Laub, 1882 in Rzeszow in Galizien geboren, hatte 1906 bei Wilhelm Wien in Würzburg mit einer Arbeit «Über sekundäre Kathodenstrahlen » promoviert und Einstein 1907 für längere Zeit in Bern besucht ${ }^{7} 1908$ war er Assistent bei Lenard geworden, und in seinen Briefen an Laub hat Einstein gelegentlich Bemerkungen über dessen neuen Chef eingestreut.

In einem undatierten Brief aus dem Jahre 1908 gratuliert Einstein zu Laubs neuer Stellung. Der Brief beginnt:

Lieber Herr Laub!

Bern Montag

Zuerst meine herzlichste Gratulation wegen der Assistentur und des damit verbundenen Einkommens. Ich hatte mein grosses Vergnügen an dieser Nachricht. Aber ich glaube, dass die Gelegenheit, mit Lenard zusammen zu arbeiten, noch weit mehr ist als Assistentur und Einkommen zusammen! Ertragen Sie Lenards Schrullen, soviel er nur haben mag. Er ist ein großer Meister, ein origineller Kopf! Vielleicht ist er ganz gut umgänglich einem Mann gegenüber, den er achten gelernt hat.

Auch in seinen Brief an Laub vom 16.3.1910 läßt Einstein eine anerkennende Bemerkung über Lenard einfließen:

Und doch müssen Sie sich glücklich preisen, dass Sie bei Lenard sind, zumal Sie ja - wie es scheint - ihn mit großem Geschick zu behandeln verstehen. Er ist nicht nur ein geschickter Meister in seiner Zunft, sondern wirklich ein Genie.

Laub hat später berichtet, daß auch Lenard in jener Zeit Einstein sehr schätzte, vor allem wegen seiner Arbeit über den lichtelektrischen Effekt ${ }^{8}$. Doch auch gegenüber der speziellen Relativitätstheorie, mit der sich sein Assistent laufend beschäftigte, war Lenard nicht voreingenommen. 1909 legte er der Heidelberger Akademie der Wissenschaften eine Arbeit Laubs vor, in der umfangreiche relativistische Rechnungen angestellt werden ${ }^{9}$, und 1910 verfaßte Laub mit Wissen und Billigung Lenards einen Bericht «Über die experimentellen Grundlagen des Relativitätsprinzips» für das von Johannes Stark herausgegebene «Jahrbuch der Radioaktivität und Elektronik». ${ }^{10}$

Erst im Juni 1909 antwortete Lenard auf Einsteins Brief mit dem folgenden Schreiben:

Hochgeehrter Herr College!

Heidelberg, 5. Juni 09

Lassen Sie mich Thnen danken für die freundlichen Zeilen anlässlich meiner letzten Sendung. Was kann mich auch mehr freuen, als wenn ein tiefer, umfassender Denker einigen Gefallen an meiner Arbeit findet. Bei dieser Gelegenheit muss ich Ihnen auch sagen, dass Ihr wertes Schreiben v. 16. Nov. 1905 seit damals immer auf meinem Schreibtisch liegt, erst in Kiel, jetzt hier, und dass ich mir immer mehr Gedanken mache über unsere verschiedenen Auffassungen über die lichtelektrischen Geschwindigkeiten und was damit 
zusammenhängt. Ich glaube nämlich, wir haben beide in gewissem Sinne recht; aber zufrieden werde ich erst sein, wenn ich sehe, wie die umfassenden, wunderbaren, von Ihnen gefundenen Beziehungen zu allem Übrigen, was ich mir vorstelle in ein Ganzes passen. Die Phosphore und deren Vorgänge geben mir fortwährend in der letzten Zeit besonderen Anlaß, an derlei zu denken. Bin ich fertig damit, so lasse ich es Sie wissen eher konnte Ihr so wertes Schreiben also noch keinen Widerhall finden. Also nicht aus Mangel war es, wie Sie sehen, dass ich immer nicht schrieb.

Mit vorzüglicher Hochachtung Ihr ergebener P. Lenard

Vielleicht verschafft die grössere Nähe Ihres Wohnsitzes künftig mir das Vergnügen, Sie einmal hier zu sehen.

Bei den «verschiedenen Auffassungen über die lichtelektrischen Geschwindigkeiten », auf die Lenard hier anspielt, handelt es sich um folgendes. Einstein vertrat die «Lichtquantenhypothese»: ein Lichtquant löst jeweils ein Elektron aus; die Energie des Elektrons hängt also von der Quantenenergie des Lichtes ab, d.h. sie ist der Frequenz proportional. Lenard war dagegen der Meinung, es handle sich beim lichtelektrischen Effekt um eine Resonanzerscheinung, für deren Erklärung nur «die Annahme complicirterer Bewegungsbedingungen der inneren Teile des Körpers» übrigbleibe, und er vermutete, «da $\beta$ die Anfangsgeschwindigkeiten der ausgestrahlten Quanten überhaupt nicht der Lichtenergie entstammen, sondern innerhalb der Atome schon vor der Belichtung vorhandenen heftigen Bewegungen, sodass die Resonanzbewegungen nur eine auslösende Rolle spielen ». ${ }^{11}$

Schon bei dieser ersten fachlichen Kontroverse zwischen Einstein und Lenard zeigte sich der Gegensatz im physikalischen Denken, der bei der dann folgenden Auseinandersetzung um die Relativitätstheorie immer deutlicher wurde. Bei der Deutung neuer, klassisch nicht ohne weiteres verständlicher Experimente war Einstein eher bereit, mit traditionellen Vorstellungen zu brechen, wenn dadurch eine einfache Erklärung möglich wurde, während Lenard dazu neigte, komplizierte, noch unerforschte, aber im Prinzip klassisch verstehbare Zusammenhänge anzunehmen.

Besonders hervorgehoben werden muß, daß sich das Verhältnis zwischen Lenard und Einstein zu diesem Zeitpunkt noch durch persönliche Hochachtung und Respekt vor der fachlichen Leistung des anderen auszeichnete. Einsteins Bemerkungen über Lenards Schrullen, die er in privaten Briefen an seinen Freund Laub äußerte, waren sicher nicht bösartig, hat er doch gleichzeitig immer wieder betont, wie hoch er Lenard in fachlicher Hinsicht einschätzte: ein großer Meister, ein origineller Kopf, wirklich ein Genie. Bei Lenard war diese Einstellung noch 1913 vorhanden, als er gegenüber Sommerfeld den Plan äußerte, in Heidelberg 
eine ordentliche Professur für theoretische Physik zu errichten, «wenn eine Persönlichkeit wie Einstein (...) dafür zur Verfügung stünde». ${ }^{12}$

Eine Wende im Verhältnis der beiden Gelehrten trat im Jahre 1910 ein: es begann die Auseinandersetzung um die Relativitätstheorie. Am 4. Juni 1910 hielt Lenard vor der Heidelberger Akademie der Wissenschaften einen Vortrag «Über Äther und Materie», der bald darauf in erster und 1911 in zweiter Auflage im Druck erschien ${ }^{13}$.

Lenard plädiert hier für die Beibehaltung der Äthervorstellung in der Physik. Dabei erkennt er «diejenigen Tatsachen (...), welche man heute unter dem Namen des Relativitätsprinzipes zusammenfaßt », ${ }^{14}$ d.h. die spezielle Relativitätstheorie, ohne weiteres an, und er sieht auch die Schwierigkeiten, die durch sie für die traditionelle Vorstellung vom Äther als dem Medium der optischen und elektrischen Erscheinungen entstanden sind. Er ist aber zuversichtlich, daß sich diese Schwierigkeiten durch Korrekturen an der bisherigen Äthervorstellung beheben lassen. Ehe man das Bild vom Äther und damit die Vorstellung von der mechanischen Begreifbarkeit der Natur aufgibt, so schreibt er, sei zu erwägen, ob wir nicht «um die Mechanik des Äthers klar zu haben, neben oder hinter dem Äther und seinen Teilen noch einen anderen Äther sollten einführen müssen ». ${ }^{15}$

Als Einstein von dem Vortrag erfuhr, schrieb er an Laub, was er davon hielt. Über den Mann, den er kurz vorher noch als Genie bezeichnet hatte, äußerte er nun:

Lenard muß aber in vielen Dingen sehr «schief gewickelt» sein. Sein Vortrag von neulich über die abstruse Ätherei erscheint mir fast infantil ${ }^{16}$.

In den folgenden sieben Jahren gab es zwischen Einstein und Lenard keinerlei Kontakt. Laub hatte Lenards Institut 1911 verlassen und war einem Ruf als Professor für theoretische Physik an die Universität La Plata (Argentinien) gefolgt. Lenard setzte in Heidelberg seine zahlreichen experimentellen Arbeiten fort, und Einstein, der 1909 die Stelle am Berner Patentamt zugunsten eines Extraordinariats in Zürich aufgegeben hatte, wurde 1911 Ordinarius in Prag und kam schließlich 1914 nach Berlin. 1915/16 veröffentlichte er seine Arbeiten zur allgemeinen Relativitätstheorie. Erst diese Arbeiten veranlaßten Lenard, sich wieder dem von Einstein behandelten Themenkomplex zuzuwenden.

Der erste von Lenard mitgetragene Angriff gegen die allgemeine Relativitätstheorie erfolgte im Jahre 1917. Ein Ergebnis der allgemeinen Relativitätstheorie ist die Erklärung der Perihelbewegung des Merkur. Ernst Gehrcke, wie Lenard ein Gegner der allgemeinen Relativitätstheorie, entdeckte, daß schon 1898 von Paul Gerber in der «Zeitschrift für Mathematik und Physik» eine Formel für 
die Merkurbewegung angegeben worden war ${ }^{17}$. Eine weitere Abhandlung Gerbers, in der die Merkurbewegung mit der auch bei Einstein auftretenden Annahme verknüpft wird, daß sich die Gravitation mit Lichtgeschwindigkeit ausbreitet, war 1902 als «Programmschrift des städt. Realgymnasiums zu Stargard in Pommern» erschienen. Gehrcke veranlaßte nun, daß diese Arbeit 1917 in den «Annalen der Physik» nachgedruckt wurde ${ }^{18}$; Einstein sollte damit der Prioritätsanspruch auf die Deutung der Merkurbewegung streitig gemacht werden, und es sollte gezeigt werden, daß die Perihelbewegung auch ohne Relativitätstheorie erklärt werden konnte.

Die Rolle, die Lenard beim Nachdruck der Gerberschen Arbeit spielte, geht aus seiner Korrespondenz mit Johannes Stark hervor. Am 10. Juli 1917 schrieb er an Stark, den Herausgeber des «Jahrbuchs für Radioaktivität und Elektronik » :

Gleichzeitig möchte ich anfragen, ob ein kleiner Originalbeitrag von mir zum Jahrbuch (weniger als 1 Bogen), über Äther und Gravitation handelnd (im Anschluss an die Gerber'sche Arbeit, die auf meine Mitveranlassung hin in den Annalen erschienen ist) baldige Aufnahme finden könnte?

Stark antwortete am 14.7.1917:

Ihre Studie über Äther und Gravitation werde ich sehr gerne in das von mir herausgegebene Jahrbuch aufnehmen. Und zwar will ich sie noch in das 4. Heft dieses Jahres einschieben. (...) Daß Sie die Aufnahme der Gerber'schen Arbeit in die Annalen mitveranlaßt haben, finde ich verdienstlich. Sie ist physikalisch gut gedacht und ist mir sympathischer als so manche theoretische Arbeit unserer Tage, welche mit einer Art didaktisch-mathematischer Zauberei die Lösung schwieriger physikalischer Probleme erfolgreich vortäuscht.

Zwei Tage später, am 16.7.1917, bedankte sich Lenard, daß Stark seinen Beitrag für das «Jahrbuch» angenommen hatte, und erläuterte noch einmal, was er mit dieser Veröffentlichung bewirken wollte:

Ich glaube darin 1. dem Äther, 2. P. Gerber ein wenig zu seinem Rechte verholfen zu haben, 3. dem allg[emeinen] Rel[ativitäts-]P[rinzip] seinen Pferdefuß gezeigt zu haben, den es aber ablegen kann, 4. eine Äther-Erklärung der Gravitation gegeben zu haben, die mir gut scheint, da sie so einfach, wie von selber, zu allem passt.

Nun trat etwas ein, was Lenard nicht erwartet hatte: im nächsten Band der «Annalen der Physik» erschienen gleich zwei vernichtende Kritiken der Gerberschen Arbeit durch Hugo Seeliger und Max von Laue, in denen Gerber mathematische Fehler nachgewiesen werden ${ }^{19}$. Das veranlaßte Lenard, Stark umgehend telegraphisch zu ersuchen, seinen Beitrag zunächst zurückzuhalten. Als Erklärung schrieb er am 20. Oktober 1917: 
Ich müsste nach jener Mitteilung v[on] Seeliger nun entweder dieselbe entkräften, oder müsste aus meinem Beitrag Gerbers Lob streichen. Zu ersterem mangelt mir im Augenblick die Zeit, da ich tief in anderer Arbeit stecke, zu letzterem entschliesse ich mich nicht ohne Weiteres. Es wird also mein Beitrag jetzt liegen bleiben müssen, bis ich weiter disponiere, und ich hoffe auf Ihre Zustimmung hierzu. Es scheint übrigens doch von vornherein, dass Gerbers Arbeit nicht ernstlich falsch sein kann; denn die Ausbreitung der Grav[itation] mit Lichtgeschwindigkeit ist heutzutage doch wohl wegen des Rel[ativitäts-] Principes (in seiner ursprünglichen, wohl unzweifelhaft giltigen Form) nicht zu bezweifeln.

Gegen die spezielle Relativitätstheorie, «das Relativitätsprincip in seiner ursprünglichen (...) Form», hatte Lenard also zu diesem Zeitpunkt noch keine Bedenken; seine Einwände galten allein der allgemeinen Relativitätstheorie. Auch das sollte noch anders werden ${ }^{20}$.

Am 9.2.1918 schickte Lenard sein überarbeitetes Manuskript erneut an Stark. Gerbers Lob, so schrieb er dazu, sei nicht ganz verschwunden, nur eingeschränkt worden. Der Aufsatz erschien unter dem Titel «Über Relativitätsprinzip, Äther, Gravitation» in Band 15 (1918) des «Jahrbuchs der Radioaktivität und Elektronik ». ${ }^{21}$

Wie der Aufsatz von 1910 ist auch dieser Artikel frei von jeder Polemik. Die spezielle Relativitätstheorie findet Lenards uneingeschränkte Zustimmung. Er vergleicht sie in ihrer Bedeutung für die Physik mit dem Energiesatz und spricht von einem allgemeingültigen Prinzip, «das nun umgekehrt zu Schlüssen und Voraussagen mit Vertrauen benutzbar sei». ${ }^{22}$

Auch die allgemeine Relativitätstheorie lehnt Lenard nicht völlig ab; er bestreitet lediglich, daß man sie auf alle beschleunigten Bewegungen anwenden könne, und will sie nur für Beschleunigungen im Gravitationsfeld gelten lassen:

Das Prinzip muß seine Allgemeingültigkeit aufgeben und nicht mehr die «Relativität aller Bewegungen » behaupten, sondern sie auf solche Bewegungen beschränken, welche unter dem Einfluß massenproportionaler Kräfte (wie es die Gravitation ist) vor sich gehen ${ }^{23}$.

Die Arbeit Gerbers hält Lenard trotz der von Seeliger und Laue nachgewiesenen Fehler für verdienstvoll, denn der Spürsinn des Verfassers habe ihn schließlich auf ein richtiges Resultat geführt. Man sei mit ihm «auffallend streng ins Gericht gegangen », während es bei experimentellen Arbeiten, die zu brauchbaren Endresultaten führten, sehr viel milder beurteilt werde, wenn der Verfasser sein Handwerkszeug mangelhaft benutzt habe ${ }^{24}$.

Noch im selben Jahr antwortete Einstein auf Lenards Einwände ${ }^{25}$. In einem «Dialog über die Einwände gegen die Relativitätstheorie » läßt er einen «Kritikus » mit einem «Relativisten » diskutieren; dabei kann es sich der Kritikus «nicht versagen, hier einige drastische Worte zu wiederholen, die Lenard neulich über den 
Gegenstand geäußert hat ». ${ }^{26}$ Lenard antwortete darauf im März 1920 in einer erweiterten Neuauflage des Aufsatzes von 1918, der jetzt als selbständige Schrift erschien. «Was Herrn Einsteins durch einen 'Relativisten' erfolgte Äußerung zum Vorliegenden betrifft», so Lenard, «so war und ist sie nicht überzeugend für mich, bzw. sie berührt gewisse Hauptpunkte zu wenig oder gar nicht. ${ }^{27}$

Die in der Auseinandersetzung um die Relativitätstheorie vorgebrachten Argumente Lenards und Einsteins sind hinreichend bekannt. Nur ein Beispiel mag zeigen, in welcher Form der Disput geführt wurde. Lenards immer wiederkehrende Einwände gegen die allgemeine Relativitätstheorie waren die damit verbundenen «Zumutungen an den einfachen Verstand». Es sei eine besondere Eigentümlichkeit des verallgemeinerten Relativitätsprinzips, «daß man (...) doch kein einziges alltägliches Beispiel seiner Geltung angeben kann, das dem gewöhnlichen Verstande, der sich mehr von der Erfahrung an der materiellen Welt als von philosophischen Bedürfnissen geleitet fühlt, einleuchtete», und sein Eingehen auf Einsteins Äußerungen erfolge nur, «um nämlich zu zeigen, daß der Standpunkt des einfachen, gesunden Verstandes, von welchem wir hier ausgehen, seine Berechtigung behält ». ${ }^{28}$

Einstein bediente sich zur Veranschaulichung von verschiedenen Bezugssystemen bekanntlich besonders gern eines Eisenbahnzuges, und auch Lenard greift bei seinen Einwänden gegen die allgemeine Relativitätstheorie dieses Bild auf. Wir stellen die diesbezüglichen Zitate aus den genannten Veröffentlichungen der besseren Übersichtlichkeit halber in Dialogform zusammen.

\section{Lenard:}

Man lasse nun den gedachten Eisenbahnzug eine deutlich ungleichförmige Bewegung machen. Wenn hierbei durch Trägheitswirkung alles im Zuge zu Trümmern geht, während draußen alles unbeschädigt bleibt, so wird, meine ich, kein gesunder Verstand einen anderen Schluß ziehen wollen, als den, daß es eben der Zug war, der mit Ruck seine Bewegung geändert hat, und nicht die Umgebung. (...) Für die naheliegende Frage, warum denn der Kirchturm neben dem Zuge nicht umgefallen sei, wenn er mit der Umgebung den Ruck gemacht habe - warum solche Folgen des Rucks so einseitig nur im Zuge sich zeigen, während dennoch kein einseitiger Schluß auf den Sitz der Bewegungsänderung möglich sein solle - hat das Prinzip anscheinend keine den einfachen Verstand befriedigende Antwort ${ }^{29}$.

Einstein :

Wie wenig es (...) angezeigt ist, in solchen Dingen den sogenannten «gesunden Verstand» als Schiedsrichter anzurufen, zeigt folgendes Gegenbeispiel. Lenard selbst sagt, es hätten sich gegen die Gültigkeit des speziellen Relativitätsprinzips (d.h. des Relativitätsprinzips bezüglich gleichförmiger Translationsbewegung der Koordinatensysteme) bisher keine zutreffenden Einwände erheben lassen. Der gleichmäßig fahrende Zug könne eben- 
sogut als "ruhend», das Geleise samt der ganzen Gegend als «gleichförmig bewegt» angesehen werden. Wird dies der "gesunde Verstand» des Lokomotiv-Führers zulassen? Er wird einwenden, daß er doch nicht die Gegend unausgesetzt heizen und schmieren müsse, sondern die Lokomotive, und daß es dementsprechend die letztere sein müsse, in deren Bewegung sich die Wirkung seiner Arbeit zeige ${ }^{30}$.

\section{Lenard:}

Herr Einstein beruft sich auf den "gesunden Verstand» eines Lokomotivführers, der auch das ursprüngliche, einfache, auf gleichförmige Bewegung beschränkte Relativitätsprinzip für ungültig erklären würde, indem er sich sagte, «daß er die Lokomotive und nicht die Gegend heizen und schmieren müsse, und daß daher sicherlich die erstere und nicht die letztere das Bewegte sei», womit - indem wir das ursprüngliche Relativitätsprinzip als gültig ansehen - der einfache "gesunde Verstand» in der Tat als irreführend gezeigt wäre. Dabei wird aber nicht bemerkt, daß dieser «gesunde Verstand» (des eben gedachten Lokomotivführers) in Wirklichkeit keiner ist, da er bekannte Dinge übersieht, was einem gesunden, bei voller Geisteskraft und Kenntnis befindlichen Menschen nicht passieren darf. Er übersieht nämlich die einfache Tatsache, daß Heizen und Schmieren der Lokomotive auch dann nötig wären, wenn die Gegend mit dem Schienengleise das Bewegte wäre, während die Lokomotive im Raum ruhte; denn die Lokomotivräder werden in jedem Falle durch die Schwere ans Gleise gepreßt, sie müssen sich drehen, es gibt Reibungsarbeit, für die die Maschine mit dem Heizen und Schmieren aufkommen muß, so lange die Relativbewegung - ganz unabhängig von der Frage nach der Absolutbewegung - nicht zum Stillstand kommen soll. Jener gesunde Verstand dagegen, welchen wir oben gegen das verallgemeinerte Relativitätsprinzip sich sträuben sahen, übersieht keine bekannten Tatsachen, und er behält somit - gänzlich unberührt von Herrn Einsteins Widerlegung - Recht, so lange nicht eben neu zu findende Tatsachen Einfluß gewinnen, die der Verstand aufnehmen muß, wenn er gesund bleiben will ${ }^{31}$.

Eine Verständigung zwischen Lenard, dem Vertreter des «gesunden Verstandes », der «als Naturforscher nicht die mathematische Zulässigkeit oder Zweckmäßigkeit von Koordinatenwahlen untersuchen » wollte ${ }^{32}$, und Einstein, dem es gerade auf die Feststellung ankam, daß alle Bezugssysteme prinzipiell gleichwertig sind («Welche Darstellung man zu wählen hat, darüber können nur Zweckmäßigkeitsgründe, aber nicht Argumente prinzipieller Art entscheidend sein.» ${ }^{33}$ ), war offensichtlich nicht möglich. So kam es, daß Lenard klagte, Einstein sei gar nicht auf seine Entgegnung eingegangen, und die von ihm vorgebrachten «Hauptpunkte» habe er zuwenig bzw. gar nicht berührt ${ }^{34}$. Auf Lenards Ausführungen von 1920 antwortete Einstein nicht mehr in einer weiteren Veröffentlichung.

Zum endgültigen Bruch kam es im August 1920, als in Berlin die Hetze gegen Einstein ihren ersten Höhepunkt erreichte. Über diese Vorfälle ist oft berichtet worden $^{35}$, so daß wir uns hier mit einer kurzen Andeutung begnügen können. Ein Paul Weyland hatte eine «Arbeitsgemeinschaft deutscher Naturforscher zur Erhaltung reiner Wissenschaft e.V.» gegründet, die in der Philharmonie Vor- 
träge gegen die Relativitätstheorie durchführte. Auf der ersten Veranstaltung am 24.8.1920 sprachen Weyland und Gehrcke.

Die erhaltenen Briefe von und an Lenard aus dieser Zeit zeigen, daß er wahrscheinlich zu Unrecht mit Weylands Aktion in Verbindung gebracht worden ist ${ }^{36}$. Weyland hatte die Absicht, in Berlin einen Zyklus von 20 Vorträgen gegen die Relativitätstheorie durchzuführen. Ein Programm dieses Vortragszyklus ist nicht mehr aufzufinden, aber wir wissen aus mehreren Briefen Laues an Sommerfeld, daß dort neben Lummer, Glaser, Wolf, Palagyi und Krauss auch Lenard als Vortragender genannt wurde ${ }^{37}$.

Nun hat aber von den hier Genannten als einziger Ludwig Glaser im Rahmen von Weylands Kampagne tatsächlich einen Vortrag gehalten ${ }^{38}$. Krauss, der ursprünglich einen Vortrag zugesagt hatte, zog sich kurzfristig von dem Unternehmen zurück ${ }^{39}$. Wie Lummer und Palagyi auf das Programm kamen, ist unbekannt. Bei Max Wolf, dem Heidelberger Astronomen, steht fest, daß er ohne sein Zutun von Weyland auf das Programm gesetzt wurde. «Ich habe dem Herrn Weyland keinen Vortrag zugesichert und bin deshalb entsetzt, meinen Namen auf der Vortragsliste zu finden», schrieb er am 30.8.1920 an Einstein und fügte hinzu, daß er diesen ganzen Vorgang auf das schärfste verurteile.

Weyland war Anfang August 1920 in Heidelberg gewesen und hatte dort auch Lenard besucht. Wir wissen davon aus einem Brief Lenards an Stark vom 2.8.1920, in dem es heißt:

Ein Herr Weyland - sehr begeistert in unserer Richtung, zur Bekämpfung undeutscher Einflüsse - war gestern bei mir und will einen Verein «Arbeitsgemeinschaft deutscher Naturforscher zur Erhaltung reiner Wissenschaft» gründen. Ich habe ihm geraten, vor Allem mit Ihnen sich in Verbindung zu setzen, damit nicht unnötig viele Neugründungen stattfinden und keine Zersplitterung unsere Nauheimer Absichten hindert.

Von einem Vortrag Lenards in Berlin ist in dem Brief nicht die Rede, und offenbar hat Weyland auch Lenard ohne dessen Zustimmung genannt. Selbst Gehrcke, der einzige namhafte Physiker, der sich 1920 Weyland zur Verfügung stellte, hat sich bald darauf von ihm distanziert und ihn in einem Brief an Lenard vom 3.2.1921 abfällig als «eine der vielen zweifelhaften Typen, die die revolutionäre, nachkriegerische Großstadt hervorgebracht hat», bezeichnet. Diese Stelle in Gehrckes Brief hat Lenard mit der Bemerkung kommentiert: «Weyland hat sich leider wirklich als Schwindler herausgestellt! Daher wollte man mir auch unterschieben, ich hätte ihm einen Vortrag zugesagt.»

Im August 1920 jedenfalls stand Lenards Name auf Weylands Programm, und das führte bei Einstein zu einer übereilten Reaktion. Unter dem Eindruck der ersten Weylandschen Veranstaltung veröffentlichte er den Artikel «Meine Ant- 
wort. Über die anti-relativitätstheoretische G.m.b.H.» im Berliner Tageblatt vom 27.8.1920, der die folgende Bemerkung über Lenard enthält:

Als ausgesprochenen Gegner der Relativitätstheorie wüßte ich unter den Physikern von internationaler Bedeutung nur Lenard zu nennen. Ich bewundere Lenard als Meister der Experimentalphysik; in der theoretischen Physik aber hat er noch nichts geleistet, und seine Einwände gegen die allgemeine Relativitätstheorie sind von solcher Oberflächlichkeit, daß ich es bis jetzt nicht für nötig erachtet habe, ausführlich auf dieselben einzugehen.

Lenard war tief gekränkt. Er war gerade in Urlaub und erfuhr von den Berliner Vorgängen durch einen Brief von Stark, der ihm am 29.8.1920 mitteilte:

Von dem Einstein-Skandal, der sich in der letzten Zeit in Berlin und in der dortigen Presse (Berliner Tageblatt) abgespielt hat, werden Sie ja gelesen haben. Einstein hat Ihnen jede theoretische Leistung ab- und dafür Oberflächlichkeit zugesprochen.

Bei seiner Rückkehr nach Heidelberg fand Lenard bei seiner Post auch Einsteins Artikel, den ihm Gehrcke geschickt hatte. Seine Reaktion zeigt der folgende, hier ungekürzt wiedergegebene Brief an Stark:

Heidelberg, 8. Sept 20.

Lieber Kollege!

Mein früherer Brief, vom Plättig, wird wohl zu Ihnen gekommen sein. Ich habe jetzt, bei meiner Rückkehr nach Heidelberg auch den Artikel von Einstein im «B[erliner] T[ageblatt]", den Sie in Ihrem letzten Briefe mir bezeichneten, hier vorgefunden Gehrcke hat ihn mir gesandt und auch Andere - und gelesen. Ich bin erstaunt über das persönliche Moment, das die Herren Einstein und auch Laue (vorher in der «T[äglichen] Rundschau " $)^{40}$ in die Angelegenheit tragen und das sie glauben, gegen mich richten zu dürfen, der ich rein sachlich in meiner Schrift aufgetreten bin, und ausser der letzteren gar nichts kundgegeben habe, was die gegen mich aufgewandten Grobheiten der Herren entschuldigen könnte. Auch kann ich aus den mir zugegangenen Druckschriften der Herren Weyland und Gehrcke ${ }^{41}$ nichts ersehen, was etwa zu Missverständnissen in Bezug auf mich hätte Anlass geben können.

Wie die Sache nun steht, muss ich sagen, dass ich sehr bezweifele ob es richtig von mir wäre, in Nauheim bei Reformen einer Gesellschaft mitzuwirken - ihr überhaupt angehörig zu bleiben - aus deren Mitte solche Grobheiten kommen, die von angesehenen Mitgliedern der Gesellschaft anscheinend noch unterstützt werden statt dass sie öffentliche Zurückweisung fänden. Denn erstens bin ich, wenn nicht ansehnliche Mitglieder der Gesellschaft sich meiner öffentlich annehmen, nicht die geeignete Person zu wesentlicher Mitwirkung, und zweitens glaube ich auch nicht mehr an den Erfolg der Besserung der Zustände in einer Gesellschaft, die solchen Ton und solches Verfahren in Ihrer Mitte für in der Ordnung hält, indem sie nicht öffentlich widerspricht. Sind meine rein sachlichen Einwände gegen die verallg[emeinerte] R[elativitäts-] Theorie zu entkräften, so muss Herr Einstein das eben zeigen - statt unartig zu werden -; ich werde mich dann freuen, nicht nur mir, sondern unzweifelhaft auch vielen anderen physikalisch Denkenden Befriedigung verschafft zu haben durch Rückhaltloses Aussprechen der Einwände. 
Kurzum, ich habe nicht die mindeste Lust, in Herrn Einsteins Gesellschaft mich zu begeben - zumal, wie gesagt, ich jetzt keinen Zweck mehr davon sehe, der das Opfer rechtfertigte - wenn nicht sichersteht, und zwar öffentlich, dass ich dabei nicht nutzlose Zielscheibe, sondern Teil eines Ganzen bin, das nur zusammen steht oder fällt. Als solches Ganzes kann nicht Herrn Weylands Arbeitsgemeinschaft dienen, weil deren Betriebsart zwar wohl gerechtfertigt aber meinem Wesen zu fremd ist; Ihre Fachgemeinschaft könnte es aber, glaube ich, wohl sein.

Mit herzlichen Grüßen Ihr ergebener P. Lenard.

Vorsitzender der Deutschen Physikalischen Gesellschaft war zu diesem Zeitpunkt Arnold Sommerfeld. Er hatte sofort erkannt, welche Gefahr es für die Gesellschaft bedeuten mußte, wenn sich zwei ihrer angesehensten Mitglieder in einen so heftigen, vor aller Öffentlichkeit geführten Kampf einließen, und unternahm einen letzten Versuch, Einstein und Lenard zu versöhnen.

Das schien auch nicht aussichtslos zu sein, da Einstein inzwischen eingesehen hatte, daß sein Artikel im «Berliner Tageblatt» ein Fehler war. Als Born ihn auf die "leider sehr ungeschickte Antwort in der Zeitung " ${ }^{42}$ hinwies, antwortete ihm Einstein: «Jeder muß am Altar der Dummheit von Zeit zu Zeit sein Opfer darbringen. (...) Und ich tat es gründlich mit meinem Artikel. ${ }^{43}$ Auch an Sommerfeld schrieb er : «Den Artikel hätte ich vielleicht nicht schreiben sollen. » ${ }^{44}$

Sommerfeld wandte sich daraufhin an beide Kontrahenten. Einstein schlug er vor, «ein Wort der Begütigung an Lenard zu schreiben. (...) Wenn Sie ihm sagen, daß Ihre Abwehr nicht dem gelehrten Kritiker, sondern dem vermeintlichen Kampfgenossen Weylands galt und daß Sie dies auf Wunsch vor der Öffentlichkeit erklären würden, besänftigt sich wohl sein Zorn. » ${ }^{45}$

Sommerfelds Brief an Lenard ist verschollen; Lenards Antwort läßt jedoch erkennen, wie sich Sommerfeld etwa geäußert haben dürfte. Sie hat folgenden Wortlaut:

Hochgeehrter Herr Kollege!

Heidelberg 14. Sept 20.

Auf die sehr werte Zuschrift v. 11 teile ich ergebenst folgendes mit.

Den Gedanken, eine Entschuldigung des Herrn Einstein mir gegenüber, noch dazu unter Voraussetzung einer ihm genehmen Äußerung meinerseits, für zufriedenstellend zu halten, muss ich mit Entrüstung zurückweisen. Denn:

1. Ob die von Herrn Einstein über mich getanen Äusserungen richtig sind, oder ob nicht, ist gänzlich unabhängig von der Frage ob ich vorhatte oder vorhabe in Berlin oder sonstwo einen Vortrag über das Rel[ativitäts]-Prinzip zu halten und in welchem Sinne dies geschähe. Diese Äusserungen des Herrn Einstein (an 3 Stellen des Artikels) schreiben mir Eigenschaften zu, die mich in den Augen der Leser herabsetzen müssen; sie sind jedenfalls ein Zeichen persönlicher Geringschätzung des Herrn Einstein gegen mich, deren 
Verwandlung in die erforderliche Hochachtung aufgrund irgend einer Zusicherung von mir höchst verwunderlich wäre.

2. Findet Herr Einstein seine Äusserungen für bereuenswert, also wohl für falsch, so hat er sie ebenso öffentlich, als sie getan worden sind, wieder zurückzuziehen; anders könnte er das an mir getane Unrecht nicht wieder gut machen - soweit das überhaupt möglich ist.

Die öffentliche Abgabe solcher Werturteile über einen Kollegen, wie von den Herren Laue «Tägl[iche] Rundschau» und Einstein geschehen, ist meinem Gefühl nach eine ganz ungehörige Überheblichkeit und bezeichnet einen Tiefstand der Gesinnung, welchen ich innerhalb der "D[eutschen] Phys[ikalischen] Ges[ellschaft]», als deren wichtige Mitglieder die genannten Herren als Berliner Vertreter der Physik wohl gelten, doch nicht vermutet hätte, obgleich ich sie für sehr reformbedürftig auch vorher schon gehalten habe.

Mit vorzüglicher Hochachtung Ihr ergebener Lenard.

Die von Lenard verlangte öffentliche Rücknahme von Einsteins Äußerungen über ihn ist nicht erfolgt. Damit war Sommerfelds Vermittlungsversuch gescheitert, und die Nauheimer Naturforscherversammlung vom 24.9.1920 führte zu dem «Zweikampf zwischen Lenard und Einstein», über den nicht nur die Fach-, sondern auch die Tagespresse ausführlich berichtet hat ${ }^{46}$.

Erst nach dieser Konfrontation, die die einzige persönliche Begegnung zwischen den beiden Physikern blieb, spielte bei Lenards Kampf gegen Einstein auch der Antisemitismus, von dem bis zu diesem Zeitpunkt nichts zu spüren war, eine zunehmende Rolle ${ }^{47}$.

\section{Anmerkungen}

1 Z.B. Armin Hermann, Die Jahrhundertwissenschaft, Stuttgart 1977, S. 40 ff.; Banesh Hoffmann und Helen Dukas, Albert Einstein, creator and rebel, London 1972, S.143f.

2 Ein Hinweis auf Lenards Brief an Einstein findet sich bei Hoffmann/Dukas, a. a. O. S. 143.

3 Annalen der Physik, 4. Folge, 17 (1905), S.132-148. Hier S.147.

4 S. 197-247.

5 Vgl. dazu John L. Heilbron, $A$ history of the problem of atomic structure from the discovery of the electron to the beginning of quantum mechanics, Diss. Berkeley 1964. Insbesondere S. $176 \mathrm{ff}$.

6 Philipp Lenard, Über den elektrischen Bogen und die Spektren der Metalle. Annalen der Physik, 4. Folge, 11 (1903), S. 636-650. Hier S. 649.

7 Carl Seelig, Albert Einstein, Zürich u. a. 1954, S.120ff. Zur Herkunft Laubs vgl. den Lebenslauf in seiner Dissertation.

8 Laub an Seelig, 10.9.1959.

9 Jakob Laub, Zur Theorie der longitudinalen magnetooptischen Effekte in leuchtenden Gasen und Dämpfen. Sitzungsberichte der Heidelberger Akademie der Wissenschaften, Mathem.-naturw. Klasse, 1 (1909/10), 6. Abhandlung. 
107 (1910), S.405-4.20.

11 Philipp Lenard, Über die lichtelektrische Wirkung. Annalen der Physik, 4. Folge, 8 (1902), S. 149-198. Hier S.170.

12 Lenard an Sommerfeld, 4.9.1913.

13 Wir benutzten die 2.Aufl., Heidelberg 1911.

14 Ib. S. 45.

15 Ib. S. 51.

16 Einstein an Laub, undatiert.

17 Paul Gerber, Die räumliche und zeitliche Ausbreitung der Gravitation. Zeitschrift für Mathematik und Physik 43 (1898), S. 93-104.

18 Ders., Die Fortpflanzungsgeschwindigkeit der Gravitation. Annalen der Physik, 4. Folge, 52 (1917), S.415-441.

19 Hugo Seeliger, Bemerkungen zu P. Gerbers Aufsatz: «Die Fortpflanzungsgeschwindigkeit der Gravitation ». Annalen der Physik, 4. Folge, 52 (1917), S. 31-32; Max von Laue, Die Fortpflanzungsgeschwindigkeit der Gravitation. Bemerkungen zur gleichnamigen Abhandlung von P. Gerber. Ib. S. 214-216.

${ }^{20}$ Lenards erste ablehnende Äußerung gegenüber der speziellen Relativitätstheorie findet sich in dem Artikel: «Über Äther und Uräther». Jahrbuch der Radioaktivität und Elektronik 17 (1920), S. 307-356. Hier S. 309.

21 S. 117-136.

22 Ib. S. 120.

23 Ib. S. 124.

24 Ib. S. 117 f.

25 Albert Einstein, Dialog über Einwände gegen die Relativitätstheorie. Die Naturwissenschaften 6 (1918), S.697-702.

26 Ib. S. 700.

${ }^{27}$ Philipp Lenard, Über Relativitätsprinzip, Äther, Gravitation, 3.Aufl., Leipzig 1921. Vorwort zur 2.Aufl., S. 9.

${ }^{28}$ Ib. S.13, S. 9.

${ }^{29}$ Ib. S.15.

30 Einstein, a.a. O. S. 701.

31 Lenard, Über Relativitätsprinzip ..., a.a.O. (Anm. 27) S. 23.

32 Ib. S. 15.

33 Einstein, a.a. O. S. 701.

${ }^{34}$ Lenard, Über Relativitätsprinzip ..., a.a. O. S. 9.

${ }^{35}$ Z.B. Armin Hermann, Der Kampf um die Relativitätstheorie. Bild der Wissenschaft 14 (1977), Heft 9, S.108-116.

36 Ib. S. 114.

$37 \mathrm{Ib}$.

38 Die Einstein-Kampagne. Berliner Tageblatt vom 3.9.1920, Abendausgabe.

39 Berliner Tageblatt vom 10.9.1920, Morgenausgabe.

40 Max von Laue, Zur Erörterung über die Relativitätstheorie. Tägliche Rundschau vom 11.8.1920, Unterhaltungsbeilage.

41 Es handelt sich wahrscheinlich um die von Weyland 1920 im Selbstverlag herausgegebenen Schriften aus dem Verlage der Arbeitsgemeinschaft deutscher Naturforscher zur 
Erhaltung reiner Wissenschaft. (Heft 1: Ernst Gehrcke, Die Relativitätstheorie, eine wissenschaftliche Massensuggestion; Heft 2: Paul Weyland, Betrachtungen über Einsteins Relativitätstheorie und die Art ihrer Einführung. Vortrag, gehalten am 24. August 1920 im großen Saal der Philharmonie zu Berlin.)

42 Born an Einstein, 8.9.1920. In: Albert Einstein/Hedwig und Max Born, Briefwechsel 1916-1955, München 1969.

43 Einstein an Born, 9.9.1920.

44 Einstein an Sommerfeld, 6.9.1920. In: Albert Einstein/Arnold Sommerfeld, Briefwechsel. Hrsg. und kommentiert von Armin Hermann, Basel 1968.

45 Sommerfeld an Einstein, 11.9.1920.

46 Hermann Weyl, Die Relativitätstheorie auf der Naturforscherversammlung in Bad Nauheim. Jahresbericht der deutschen Mathematiker-Vereinigung 31 (1922), S. 51-63; Berliner Tageblatt vom 24.9.1920, Abendausgabe.

47 Hier ist vor allem das «Mahnwort an deutsche Naturforscher» zu nennen, das Lenard 1922 der 2. Auflage seiner Schrift «Über Äther und Uräther» voranstellte.

\section{Nachweis der ungedruckten Briefe}

Lenard an Einstein, Wolf an Einstein: Estate of Albert Einstein (New York). Einstein an Laub, Laub an Seelig: ETH-Bibliothek Zürich. (Die Originale der Briefe Einsteins sind verschollen. Es handelt sich um Abschriften, die Laub für den Einstein-Biographen Carl Seelig angefertigt hat.) Lenard an Sommerfeld: Deutsches Museum München.

Die Briefe Lenards an Stark, der Brief Einsteins an Lenard und der Brief Gehrckes an Lenard sind in Privatbesitz. Wir danken den Besitzern, daß sie uns Ablichtungen überlassen haben. Für die Genehmigung zur Veröffentlichung der Briefe Lenards danken wir Frau Gisela Sievert. Die Veröffentlichung der Briefe Einsteins erfolgt mit Genehmigung des «Estate of Albert Einstein» (New York), die des Zitats aus dem Brief von Max Wolf mit Erlaubnis von Prof. Dr. Franz Wolf.

Herr Otto Nathan (Estate of Albert Einstein) und Herr Alvin E. Jaeggli (ETH-Bibliothek Zürich) waren behilflich bei der Zusammenstellung der von uns benutzten Briefe von und an Einstein. Fräulein Traute Braun überließ uns die für ihre Magisterarbeit gesammelten Ausschnitte aus dem Berliner Tageblatt und der Täglichen Rundschau. Dr. David Cassidy half bei der Anfertigung der englischen Zusammenfassung. Ihnen allen ein herzliches Dankeschön!

\section{Summary}

The publication of Einstein's paper on the photoelectric effect in 1905 brought him to Lenard's attention. A brief exchange of letters took place in which interpretations of spectral lines and the photoelectric effect were discussed. These letters and those from Einstein to Lenard's assistant Jakob Laub show that Lenard and Einstein possessed a high admiration and respect for one another. Einstein called Lenard a great master and genius; Lenard wanted to appoint a personality like Einstein as professor in Heidelberg as late as 1913. 
The relationship between the two physicists took a turn for the worse when Lenard began studying the theory of relativity. In 1910 he pleaded for a retention of the ether in a lecture which Einstein described as infantile. After 1917 he opposed the general theory of relativity. In 1918 he published his objections in a treatise "On the Relativity Principle, Ether and Gravitation". At that time Lenard still accepted the special theory of relativity and wanted only to modify the general theory in part. Einstein answered Lenard's objections, Lenard responded anew, but in these publications the personal side of the conflict moved more and more into the foreground. The final break came in 1920 when a certain Paul Weyland organized an anti-Semitic campaign against Einstein and his physics in Berlin in which Lenard's name was used. From Lenard's correspondence with Stark and Gehrcke it is clear that Weyland had misused Lenard's name. Einstein, who did not know this, attacked Lenard in a newspaper article. Lenard was deeply wounded and demanded a public apology. An attempt by Sommerfeld to reconcile the two failed. In September 1920 Einstein and Lenard squared off during a heated discussion in Bad Nauheim, and from then on Lenard's personal anti-Semitism began playing an increasingly central role in his opposition to relativity theory and its founder.

Prof. Dr. Andreas Kleinert

Friedenstraße 20

D - 7142 Marbach/N.
Dr. Charlotte Schönbeck

Silbergasse 45

D-6901 Wilhelmsfeld 